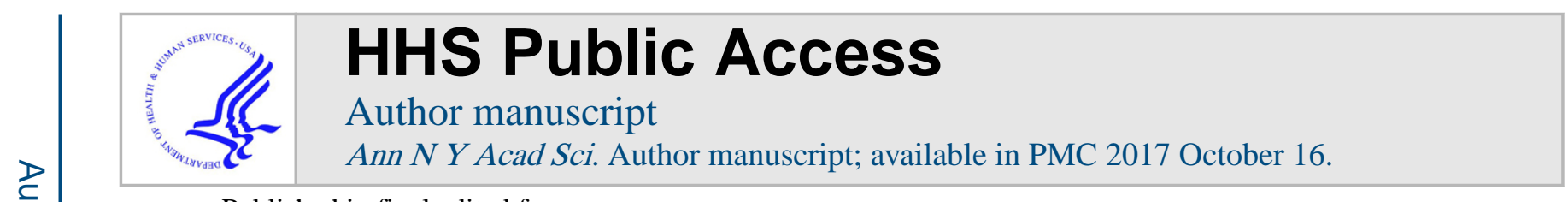

Published in final edited form as:

Ann N Y Acad Sci. 2010 June ; 1198: 173-181. doi:10.1111/j.1749-6632.2009.05429.x.

\title{
Bone cancer pain
}

Juan Miguel Jimenez-Andrade ${ }^{1}$, William G. Mantyh ${ }^{1}$, Aaron P. Bloom ${ }^{1}$, Alice S. Ferng ${ }^{2}$, Christopher P. Geffre ${ }^{2}$, and Patrick W. Mantyh ${ }^{1,3,4}$

${ }^{1}$ Department of Pharmacology, University of Arizona, Tucson, Arizona

${ }^{2}$ Department of Physiological Sciences, College of Medicine University of Arizona, Tucson, Arizona

${ }^{3}$ Arizona Cancer Center, University of Arizona, Tucson, Arizona

${ }^{4}$ Research Service, VA Medical Center, Minneapolis, Minnesota

\section{Abstract}

In the United States, cancer is the second most common cause of death and it is expected that about 562,340 Americans will have died of cancer in 2009. Bone cancer pain is common in patients with advanced breast, prostate, and lung cancer as these tumors have a remarkable affinity to metastasize to bone. Once tumors metastasize to bone, they are a major cause of morbidity and mortality as the tumor induces significant skeletal remodeling, fractures, pain, and anemia. Currently, the factors that drive cancer pain are poorly understood. However, several recently introduced models of bone cancer pain, which closely mirror the human condition, are providing insight into the mechanisms that drive bone cancer pain and guide the development of mechanismbased therapies to treat the cancer pain. Several of these mechanism-based therapies have now entered human clinical trials. If successful, these therapies have the potential to significantly enlarge the repertoire of modalities that can be used to treat bone cancer pain and improve the quality of life, functional status, and survival of patients with bone cancer.

\section{Keywords}

osteosarcoma; prostate; therapies

\section{Bone cancer pain in humans}

The majority of patients with metastatic bone disease experience moderate to severe pain and bone pain is one of the most common types of chronic pain in these patients. ${ }^{1}$ Although bone is not a vital organ, many common tumors (breast, prostate, thyroid, kidney, and lung) have a strong predilection to simultaneously metastasize to multiple bones. ${ }^{1,2}$ It has been reported that tumor mestastases to the skeleton affect over 400,000 individuals in the United

Address for correspondence: Patrick W. Mantyh, Ph.D., Department of Pharmacology, College of Medicine, University of Arizona, 1656 E. Mabel, Rm. \#119, PO Box 245215, Tucson, AZ 85724. Voice: 520-626-0742; fax: 520-626-4182. pmantyh@email.arizona.edu.

Conflicts of interest

The authors declare no conflicts of interest. 
States annually. Tumor growth in bone results in pain, hypercalcemia, anemia, increased susceptibility to infection, skeletal fractures, compression of the spinal cord, spinal instability, and decreased mobility, all of which compromise the patient's functional status, quality of life, and survival. ${ }^{1,3}$ Once tumor cells have metastasized to the skeleton, tumorinduced bone pain is usually described as dull in character, constant in presentation, and gradually increasing in intensity with time. ${ }^{3}$ Adherence to the World Health Organization analgesic ladder, along with adjuvant therapies such as bisphosphonates, corticosteroids, radiotherapy, and radionucleotides, can frequently control ongoing bone cancer pain. However, both opiates and nonsteroidal anti-inflammatory drugs may have significant doselimiting side effects.

As tumor growth and tumor-induced bone remodeling progress, severe "incident pain" frequently occurs. ${ }^{3}$ Incident pain is also known as "breakthrough pain" as the pain breaks through the analgesic regime that is controlling the ongoing pain. This pain is defined as an intermittent episode of extreme pain which occurs spontaneously, during non-noxious movement, ${ }^{4}$ or mechanical loading of the tumor-bearing bone(s). A major problem with incident pain in bone cancer is that it is usually more severe than ongoing pain, it appears suddenly (within seconds to minutes) and it can occur multiple times each day. ${ }^{4}$ Given the therapies that are currently available, as well as the rapid onset and severity of incident pain, this pain remains one of the most challenging of cancer pains to fully control. ${ }^{4}$ When present, incident pain can be highly debilitating to the patient's functional status and quality of life. ${ }^{2-4}$

\section{Preclinical models of bone cancer pain}

Previously, there were two commonly used in vivo mouse models to study tumor-induced bone destruction. In the first model, tumor cells are injected into the left ventricle of the heart and then spread to multiple sites including the bone marrow where they grow and induce remodeling of the surrounding bone. ${ }^{5,6}$ While this model replicates the observation that most tumor cells metastasize to multiple sites including bone, a major problem with this model is the animal-to-animal variability in the sites, size, and extent of the metastasis. Because the tumors frequently metastasize to vital organs such as the lung or liver, the general health of the animal is also variable, making behavioral assessment of bone pain difficult. Given these problems, intracardiac injection of cancer cells as a model for bone cancer pain has proven difficult.

The second major model used to study tumor-induced bone destruction involves the direct injection of osteolytic sarcoma cells into the intramedullary space of the mouse tibia or femur (Fig. 1A). A major advance leading to the currently used model was to plug the injection hole with a dental amalgam or bone cement (Fig. 1B), which by tightly binding and sealing the injection hole, confining tumor cells to the marrow space of the bone and prevents tumor invasion into surrounding soft tissue (Fig. 1C). ${ }^{7}$

Mouse osteosarcoma tumor cells were the first cell type that was extensively used in this model (Fig. 1C). These cells were injected and confined to the intramedullary space of the mouse femur (Fig. 1C). ${ }^{8}$ The tumor cells grow in a highly reproducible fashion (Fig. 2E) 
and, as they proliferate, replace the hemapoetic cells that normally populate the bone marrow (Fig. 2D) ${ }^{7,8}$ Eventually, the entire marrow space is filled with tumor cells and tumor-associated inflammatory/immune cells (Fig. 2E). ${ }^{7,8}$ In terms of bone remodeling, injection of osteosarcoma cells into the femur induces a dramatic proliferation and hypertrophy of osteoclasts at the tumor-bone interface, as well as significant bone destruction in both the proximal and distal heads of the femur (Figs. $2 \mathrm{~B}$ and E). ${ }^{7}$ In this model, ongoing and movement-evoked pain behaviors increase in severity with time and are correlated with the tumor growth and progressive tumor-induced bone destruction, ${ }^{7,8}$ which mirrors what occurs in patients with primary or metastatic bone cancer. Although osteosarcoma cells were the first cells used in this model, other animal and human tumor cells, including prostate, breast, melanoma, colon, and lung tumors, have now been used in the closed femur model of bone cancer pain. ${ }^{9}$

Prostate cancer is unique in that bone is often the only clinically detectable site of metastasis. Prostate tumors that have metastasized to bone frequently induce bone pain, which can be difficult to fully control as it seems to be driven simultaneously by inflammatory, neuropathic, and tumorigenic mechanisms. In an effort to develop mechanism-based therapies that attenuate bone cancer pain due to other metastatic tumors, such as those from the prostate, we recently characterized another bone cancer pain model ${ }^{10}$ (Figs. 2C and F). This model utilizes canine prostate tumor cells (ACE-1) that are injected and confined to the intramedullary space in the femur of nude mice. ${ }^{10}$ Using this model it was shown that significant tumor-induced, pain-related behaviors were first observed at 9 days following injection of prostate tumor cells and continued to increase until 19 days postinjection at which time the mice were euthanized. ${ }^{10}$ Prostate tumor cells induce significant formation of new woven bone at the proximal head, distal end, and the diaphysis of the bone (Figs. 2C and F). The marked bone formation induced by prostate cancer cells is also accompanied by bone destruction, giving the tumor-bearing femur a unique scalloped appearance when assessed by micro-computed tomography ( $\mu \mathrm{CT}$ ) (Fig. 2C) or with traditional histological methodology (Fig. 2F). This appearance is consistent with what is observed in human patients with prostate tumor metastases.

\section{Tumor and osteoclast-induced acidosis and bone cancer pain}

Studies in both humans and animals have suggested that osteoclasts (the cells that break down bone) play a significant role in cancer-induced bone loss ${ }^{11}$ and that osteoclasts contribute to the etiology of bone cancer pain. ${ }^{12,13}$ Osteoclasts are terminally differentiated, multinucleated, monocyte lineage cells that resorb bone by maintaining an extracellular microenvironment of acidic $\mathrm{pH}(4.0-5.0)$ at the osteoclast-mineralized bone interface (Fig. 3) ${ }^{14}$ Both osteolytic (bone destroying) and osteoblastic (bone forming) cancers are characterized by osteoclast proliferation and hypertrophy. ${ }^{7,15,16}$ Thus, osteoclast-mediated bone remodeling results in robust production of extracellular protons, ${ }^{17}$ which are known to be potent activators of nociceptors (Fig. 3B). ${ }^{18}$ This raises the possibility that the acidic microenvironment produced by osteoclasts contributes significantly to bone cancerassociated pain through activation of acid-sensitive nociceptors that innervate the marrow, mineralized bone, and periosteum. ${ }^{19}$ 
The finding that sensory neurons can be directly excited by protons originating from cells, such as osteoclasts in bone, has generated clinical interest in targeting acid sensing channels expressed by nociceptors. Studies have shown that subsets of sensory neurons express different acid-sensing ion channels. ${ }^{18}$ Two acid-sensing ion channels expressed by nociceptors are transient receptor potential vanilloid 1 (TRPV1) and acid-sensing ion channel-3 (ASIC-3) (Fig. 3B). ${ }^{18}$ Both of these channels are sensitized and excited by a decrease in $\mathrm{pH}$ in the range of 4.0-5.0, which is generated by osteoclasts (Fig. 3B). ${ }^{18} \mathrm{In}$ addition to osteoclast-induced acidosis, the tumor stroma ${ }^{20}$ and areas of the tumor which are necrotic typically exhibit lower extracellular $\mathrm{pH}$ than surrounding normal tissues. As inflammatory and immune cells invade the tumor stroma, these cells also release protons that generate a local acidosis. ${ }^{12,19}$

Animal and clinical studies of bone cancer have reported that the antiresorptive effects of bisphosphonate therapies simultaneously reduce bone cancer pain, tumor-induced bone destruction, and tumor growth within the bone. ${ }^{12,13,21}$ Bisphosphonates are a class of antiresorptive compounds that are pyrophosphate analogues which display high affinity for calcium ions, causing them to rapidly and avidly bind to the mineralized matrix of bone. ${ }^{15}$ As osteoclasts resorb bone they use endocytosis and transcytosis to clear the bone breakdown products from the osteoclast-bone interface (including the bisphosphonate, which is bound to the mineralized bone). Bisphosphonates, once taken up by the osteoclasts, induce loss of function and ultimately apoptosis of the osteoclasts. ${ }^{15}$

It should be stressed that while bisphosphonates are approved and are frequently used to reduce tumor-induced bone destruction and bone cancer pain, bisphosphonates do have unwanted side effects (including induction of arthralgias and osteonecrosis of the jaw), ${ }^{15}$ and it has yet to be definitively shown that bisphosphonates increase the survival of patients with bone cancer. For this reason, other therapies targeting osteoclasts are already in mid- to late-stage clinical trials and hold significant promise for alleviating bone cancer pain and tumor-induced bone remodeling. One line of therapies attempts to block the binding of receptor activator for nuclear factor $\mathrm{kB}$ ligand (RANKL), which is an essential regulator of osteoclasts. ${ }^{11}$ Studies in mice have shown that sequestration of RANKL with OPG attenuates sarcoma-induced bone pain, bone remodeling, and tumor growth within the bone (Figs. 4B-E). ${ }^{7}$ Recent small clinical studies have shown that in humans with multiple myeloma or breast cancer metastasis to bone, Denosumab (a fully humanized monoclonal antibody that inhibits RANKL) markedly reduces tumor-induced bone resorption and skeletal-related events (which include fracture and pain). ${ }^{22}$ Currently, phase III clinical trials are underway for assessing Denosumab's effects on attenuating cancer-induced bone loss in breast and prostate cancers, ${ }^{23}$ skeletal related events (pain, fracture) due to the spread of cancer to the bone in multiple myeloma and multiple solid tumors, as well as the potential to delay bone metastases in prostate cancer. ${ }^{24}$

The mechanisms by which inhibition of osteoclast activity (either by bisphosphonates or OPGL/RANKL binding molecules) attenuates bone cancer pain may involve, at least in part, the reduction of osteoclast-induced acidosis. Tissue acidosis may activate nociceptors that innervate the bone through multiple mechanisms, ${ }^{12,18}$ but TRPV1 has been proposed to play a major role in acid-induced activation of nociceptors (Fig. 3B). Recent pharmacological 
studies showed that selective TRPV1 antagonists significantly decreased ongoing (JNJ-17203212, ABT-102, and SB366791) and movement-evoked (JNJ-17203212 and ABT-102) pain-related behaviors in the mouse model of bone cancer pain, without any observable behavioral side effects, such as ataxia or hypoactivity. ${ }^{19,25}$

Although the above discussion has focused on osteoclast-mediated acidosis as a mechanism that drives bone cancer pain, both osteolytic and osteoblastic tumors induce a loss of the mechanical strength and stability of the tumor-bearing bone so that normally innocuous mechanical stress can now produce distortion of the mechanosensitive sensory nerve fibers that innervate the bone (Fig. 3B). Previous results have shown that the pain associated with fracture is significantly attenuated if the bone is stabilized and returned to its normal orientation. ${ }^{26}$ Preservation of the mechanical strength of bone should reduce movementinduced incident pain, as this pain is probably driven by activation of normally silent mechanosensitive nociceptors that innervate the bone.

\section{Tumor-derived products in generation of bone cancer pain}

In most cancers, the tumor mass is composed of tumor and tumor stromal cells, the latter of which includes macrophages, neutrophils, T-lymphocytes, fibroblasts, and endothelial cells. Tumor and/or tumor stromal cells have been shown to secrete a variety of factors that sensitize or directly excite primary afferent neurons (Fig. 3). These factors include prostaglandins, bradykinin, tumor necrosis factor-alpha, endothelins, interleukins- 1 and -6 , epidermal growth factor, transforming growth factor-alpha, platelet-derived growth factor, and nerve growth factor (NGF) (Fig. 3). ${ }^{12,27}$

One tumor/stromal cell product that is of significant interest in the etiology of bone cancer pain is NGF. Previous studies have shown that NGF may directly activate sensory neurons that express the TrkA receptor and/or modulate the expression of proteins of sensory neurons expressing TrkA or p75 receptor (Fig. 3). ${ }^{28}$ Anti-NGF antibody therapy may be particularly effective in blocking bone cancer pain as NGF appears to be integrally involved in the up-regulation, sensitization, and disinhibition of multiple neurotransmitters, ion channels and receptors in the primary afferent nerve fibers that synergistically increase nociceptive signals originating from the tumor-bearing bone.

To test the hypothesis that blocking NGF from binding to its cognate receptor TrkA is efficacious in reducing bone cancer pain, the analgesic efficacy of a murine anti-NGF monoclonal antibody was evaluated in two animal models of bone cancer. ${ }^{10,29}$ These models included the primarily osteolytic mouse osteosarcoma line that expresses high levels of NGF, ${ }^{29}$ and the primarily osteoblastic canine ACE-1 prostate, where NGF expression is undetectable (Fig. 5) ${ }^{10}$ In both of these models it was demonstrated that administration of an anti-NGF antibody was efficacious in reducing both early- and late-stage bone cancer pain-related behaviors (Fig. 5) and that this reduction in pain-related behaviors was greater than that achieved with acute administration of $10 \mathrm{mg} / \mathrm{kg}$ of morphine sulfate. ${ }^{10,29}$ This data suggests that therapeutic targeting of NGF, or its cognate receptor TrkA, may be useful in blocking bone cancer pain whether or not the tumor that has metastasized to bone expresses NGF. Presumably, in the case where the tumor cells themselves do not express NGF, it is the 
tumor stromal cells that are expressing and secreting NGF, as tumor stromal cells comprise $2-60 \%$ of the total tumor mass. Currently, a fully humanized monoclonal antibody to NGF (Tanezumab) has been tested in human patients with osteoarthritis (OA) and this therapy was effective at reducing OA pain. ${ }^{30}$ Human clinical trials evaluating Tanezumab's effects at reducing bone cancer pain in patients with advanced breast or prostate cancer were scheduled to commence mid-2009. ${ }^{31}$

\section{Neuropathic component of bone cancer pain}

Because sensory and sympathetic neurons are present within the bone marrow, mineralized bone, and periosteum, and all these compartments are ultimately impacted by fractures, ischemia, or the presence of tumor cells, sensory fibers in any of these tissues may play a role in the generation and maintenance of bone cancer pain.

In examining the changes in the sensory innervation of bone that are induced by the primarily osteolytic sarcoma cells, sensory fibers were observed at and within the leading edge of the tumor in the deep stromal regions of the tumor. ${ }^{32}$ These sensory nerve fibers displayed a discontinuous and fragmented appearance, suggesting that following initial activation by the osteolytic tumor cells, the distal processes of the sensory nerve fibers were injured by the invading tumor cells. ${ }^{32}$

The tumor-induced injury and/or remodeling of sensory nerve fibers in these bone cancer pain models was also accompanied by an increase in ongoing and movement-evoked pain behaviors, an upregulation of galanin by sensory neurons that innervate the tumor-bearing femur, an upregulation of glial fibrillary acidic protein, hypertrophy of satellite cells surrounding sensory neuron cell bodies within the ipsilateral DRG, and macrophage infiltration of the DRG ipsilateral to the tumor-bearing femur. ${ }^{12}$ Similar neurochemical changes have been described following peripheral nerve injury in other noncancerous neuropathic pain states. ${ }^{33}$ In addition, chronic treatment with Gabapentin in the sarcoma model attenuated both ongoing and movement-evoked bone cancer-related pain behaviors, but did not influence tumor growth or tumor-induced bone destruction. ${ }^{32}$ In light of these findings, we can suggest that bone cancer pain is driven by a neuropathic pain component. Currently, clinical trials are underway for assessing the effects of pregabalin (structurally related to gabapentin) on attenuating chronic bone pain related to metastases. ${ }^{34}$

\section{Conclusions}

Over the last decade, progress has been made in laying the foundation for a mechanismbased understanding of the factors that drive bone cancer pain. Interestingly, several therapies that attenuate bone cancer pain may also reduce tumor growth and tumor-induced bone remodeling. Thus, bisphosphonates are commonly used to treat bone cancer pain and other therapies including Denosumab (anti-RANKL; Amgen Inc., Thousand Oaks, CA), Tanezumab (anti-NGF; Pfizer Inc., New York, NY), and Pregabalin (Pfizer) are in mid- to late-stage clinical trials. We are beginning to understand the mechanisms that drive bone cancer. If this progress can be sustained and expanded, these advances have the potential to 
enlarge the repertoire of therapies available to treat bone cancer pain and significantly improve the quality of life, functional status, and survival of affected patients.

\section{References}

1. Coleman RE. Clinical features of metastatic bone disease and risk of skeletal morbidity. Clin. Cancer Res. 2006; 12:6243s-6249s. [PubMed: 17062708]

2. Coleman RE. Skeletal complications of malignancy. Cancer. 1997; 80:1588-1594. [PubMed: 9362426]

3. Mercadante S. Malignant bone pain: pathophysiology and treatment. Pain. 1997; 69:1-18. [PubMed: 9060007]

4. Zeppetella G. Impact and management of breakthrough pain in cancer. Curr. Opin. Support Palliat. Care. 2009; 3:1-6. [PubMed: 19365156]

5. Arguello F, Baggs RB, Frantz CN. A murine model of experimental metastasis to bone and bone marrow. Cancer Res. 1988; 48:6876-6881. [PubMed: 3180096]

6. Yoneda T, Sasaki A, Mundy GR. Osteolytic bone metastasis in breast cancer. Breast Cancer Res. Treatment. 1994; 32:73-84.

7. Honore $\mathrm{P}$, et al. Osteoprotegerin blocks bone cancer-induced skeletal destruction, skeletal pain and pain-related neurochemical reorganization of the spinal cord. Nat. Med. 2000; 6:521-528. [PubMed: 10802707]

8. Schwei MJ, et al. Neurochemical and cellular reorganization of the spinal cord in a murine model of bone cancer pain. J. Neurosci. 1999; 19:10886-10897. [PubMed: 10594070]

9. Sabino MA, et al. Different tumors in bone each give rise to a distinct pattern of skeletal destruction, bone cancer-related pain behaviors and neurochemical changes in the central nervous system. Int. J. Cancer. 2003; 104:550-558. [PubMed: 12594809]

10. Halvorson KG, et al. A blocking antibody to nerve growth factor attenuates skeletal pain induced by prostate tumor cells growing in bone. Cancer Res. 2005; 65:9426-9435. [PubMed: 16230406]

11. Lipton A. Future treatment of bone metastases. Clin. Cancer Res. 2006; 12:6305s-6308s. [PubMed: 17062719]

12. Mantyh PW. Cancer pain and its impact on diagnosis, survival and quality of life. Nat. Rev. Neurosci. 2006; 7:797-809. [PubMed: 16988655]

13. von Moos R, et al. Metastatic bone pain: treatment options with an emphasis on bisphosphonates. Support Care Cancer. 2008; 16:1105-1115. [PubMed: 18682990]

14. Delaisse, JM., Vaes, G. Mechanism of mineral solubilization and matrix degradation in osteoclastic bone resorption. In: Rifkin, BR., Gay, CV., editors. Biology and Physiology of the Osteoclast. CRC; Ann Arbor: 1992. p. 289-314.

15. Drake MT, Clarke BL, Khosla S. Bisphosphonates: mechanism of action and role in clinical practice. Mayo Clin. Proc. 2008; 83:1032-1045. [PubMed: 18775204]

16. Halvorson KG, et al. Similarities and differences in tumor growth, skeletal remodeling and pain in an osteolytic and osteoblastic model of bone cancer. Clin. J. Pain. 2006; 22:587-600. [PubMed: 16926574]

17. Teitelbaum SL. Osteoclasts: what do they do and how do they do it? Am. J. Pathol. 2007; 170:427435. [PubMed: 17255310]

18. Julius D, Basbaum AI. Molecular mechanisms of nociception. Nature. 2001; 413:203-210. [PubMed: 11557989]

19. Ghilardi JR, et al. Selective blockade of the capsaicin receptor TRPV1 attenuates bone cancer pain. J. Neurosci. 2005; 25:3126-3131. [PubMed: 15788769]

20. Griffiths JR. Are cancer cells acidic? Br. J. Cancer. 1991; 64:425-427. [PubMed: 1911181]

21. Lipton A. Emerging role of bisphosphonates in the clinic - antitumor activity and prevention of metastasis to bone. Cancer Treat Rev. 2008; 34(Suppl 1):S25-S30. [PubMed: 18486347]

22. Body JJ, et al. A study of the biological receptor activator of nuclear factor-kappaB ligand inhibitor, denosumab, in patients with multiple myeloma or bone metastases from breast cancer. Clin. Cancer Res. 2006; 12:1221-1228. [PubMed: 16489077] 
23. [Accessed April 2009] http://clinicaltrials.gov. NCT00556374, NCT00321620

24. Lipton A, Jun S. RANKL inhibition in the treatment of bone metastases. Curr. Opin. Support Palliat. Care. 2008; 2:197-203. [PubMed: 18685421]

25. Niiyama Y, et al. SB366791, a TRPV1 antagonist, potentiates analgesic effects of systemic morphine in a murine model of bone cancer pain. Br. J. Anaesth. 2009; 102:251-258. [PubMed: 19038965]

26. Rubert Cynthia, HR., Martin, M. Orthopedic management of skeletal metastases. In: Body, J-J., editor. Tumor Bone Disease and Osteoporsis in Cancer Patients. Marcel Dekker; New York City: 2000. p. 305-356.

27. Joyce JA, Pollard JW. Microenvironmental regulation of metastasis. Nat. Rev. Cancer. 2009; 9:239-252. [PubMed: 19279573]

28. Pezet S, McMahon SB. Neurotrophins: mediators and modulators of pain. Annu. Rev. Neurosci. 2006; 29:507-538. [PubMed: 16776595]

29. Sevcik MA, et al. Anti-NGF therapy profoundly reduces bone cancer pain and the accompanying increase in markers of peripheral and central sensitization. Pain. 2005; 115:128-141. [PubMed: 15836976]

30. Schnitzer, TJ., et al. 12th World Congress of Pain: PT 214. Glasgow; Scotland, UK: 2008. Efficacy and safety of Tanezumab (PF04383119), an anti-nerve growth factor (NGF) antibody, for moderate to severe pain due to osteoarthritis $(\mathrm{OA})$ of the knee: a randomized trial.

31. [Accessed April 2009] http://clinicaltrials.gov. NCT00545129, NCT00830180

32. Peters CM, et al. Tumor-induced injury of primary afferent sensory nerve fibers in bone cancer pain. Exp. Neurol. 2005; 193:85-100. [PubMed: 15817267]

33. Obata K, et al. Contribution of injured and uninjured dorsal root ganglion neurons to pain behavior and the changes in gene expression following chronic constriction injury of the sciatic nerve in rats. Pain. 2003; 101:65-77. [PubMed: 12507701]

34. [Accessed April 2009] http://clinicaltrials.gov. NCT00381095 

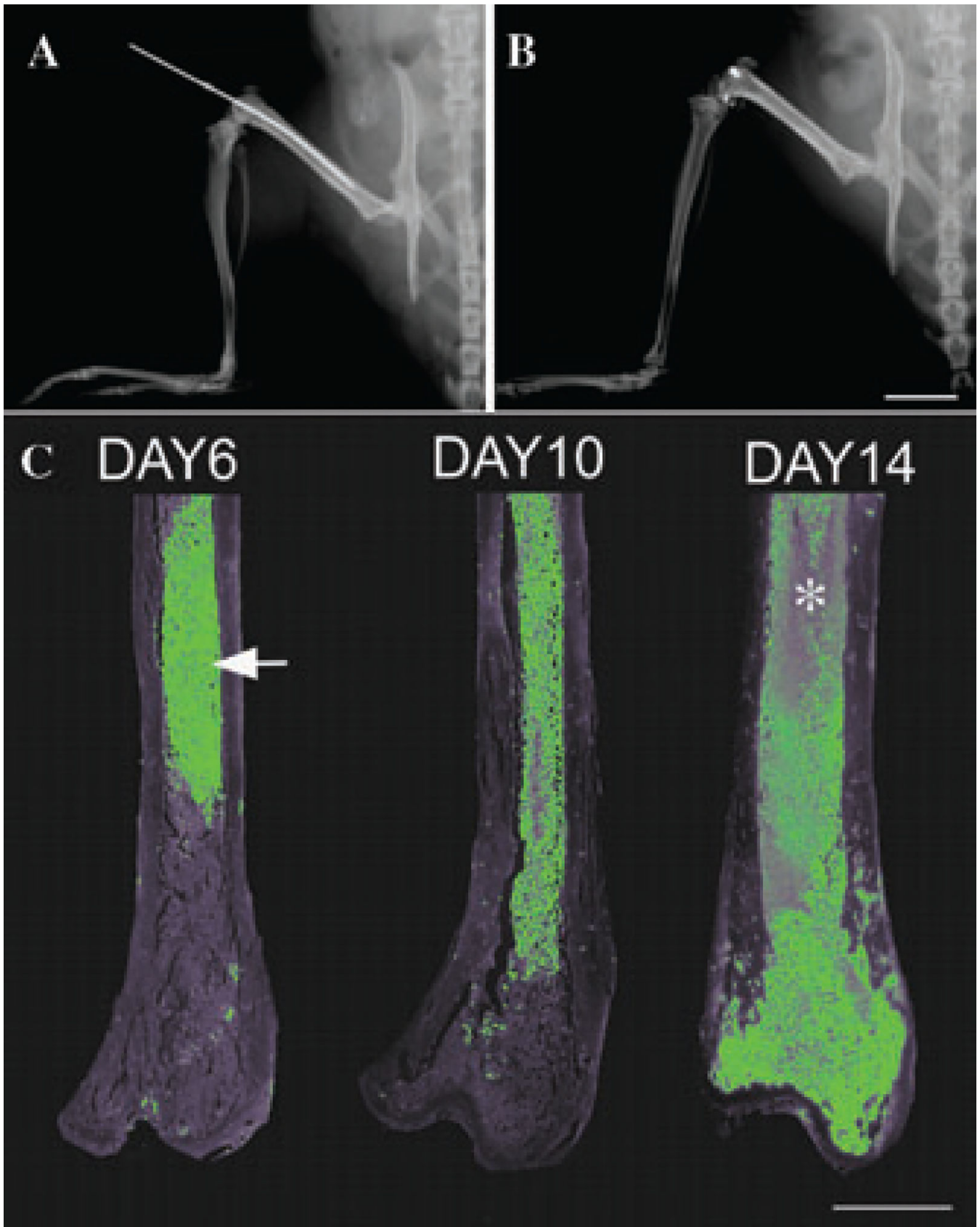

Figure 1.

Development of a mouse model of bone cancer pain and disease progression. Low-power anterior-posterior radiograph of a mouse femur showing the unilatereal injection of sarcoma cells into the femur (A) and confinement of the tumor cells in marrow space with an amalgam plug (B). The present model allows a simultaneous visualization and quantitative evaluation of the tumor burden by using 2472 sarcoma cancer cells genetically manipulated to express enhanced green fluorescent protein (GFP). (C) GFP-transfected tumor cells (green) injected into the ipsilateral femurs at day 6 (arrow), day 10, and day 14 postinjection. Scale bar: $6 \mathrm{~mm}$ in (A, B) and $1.5 \mathrm{~mm}$ in (C). 

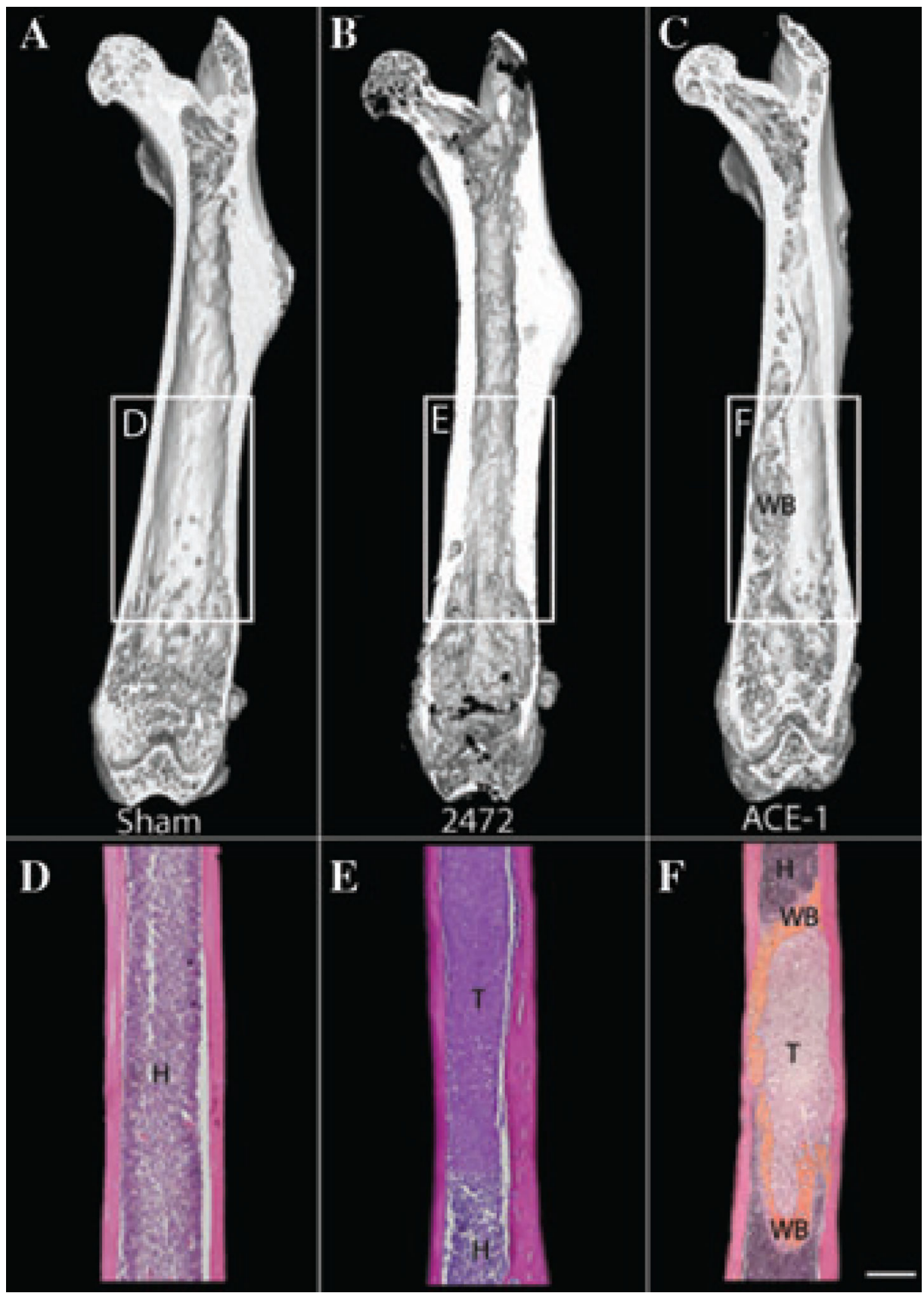

Figure 2.

Bone remodeling and tumor growth in the 2472 sarcoma and ACE-1 prostate carcinomainjected femurs have different characteristics depending on the osteolytic or osteoblastic component of the tumor cells as assessed by $\mu \mathrm{CT}$ imaging and hematoxilin and eosin (H\&E) staining. Sham-injected femurs present relative absence of bone formation or bone destruction (A, D). The 2472 sarcoma-injected femurs display a primarily osteolytic appearance visible as regions absent of trabecular bone at the proximal and distal heads (B) as well as replacement of normal hematopoietic cells by tumor cells (E). The ACE-1 prostate carcinoma-injected femurs mainly present an osteoblastic appearance which is 
characterized by pathologic bone formation in the intramedullary space (C) surrounding pockets of tumor cells which generate diaphyseal bridging structures (F). (A-F) Scale bar: $0.5 \mathrm{~mm}$. T, tumor; H, normal hematopoietic cells; WB, ACE-1-induced woven bone formation. 

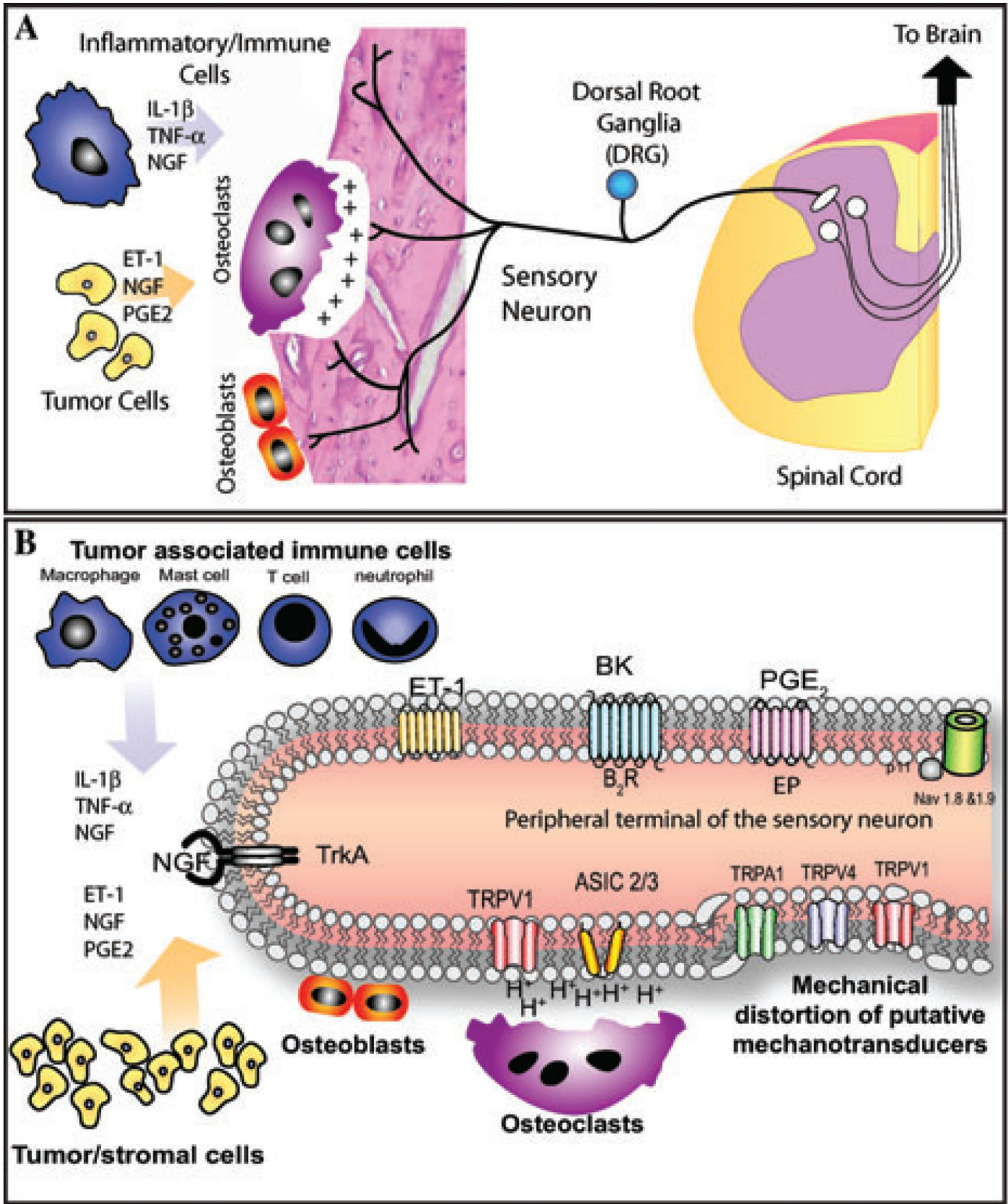

Figure 3.

Schematic showing factors in bone (A) and receptors/channels expressed by nociceptors that innervate the skeleton (B) that drive bone cancer pain. A variety of cells (tumor cells and stromal cells including inflammatory/immune cells, osteoclasts, and osteoblasts) drive bone cancer pain (A). Nociceptors that innervate the bone use several different types of receptors to detect and transmit noxious stimuli that are produced by cancer cells (yellow), tumorassociated immune cells (blue), or other aspects of the tumor microenvironment. There are multiple factors that may contribute to the pain associated with cancer (B). The transient receptor potential vanilloid receptor-1 (TRPV1) and acid sensing ion channels (ASICs) 
detect extracellular protons produced by tumor induced tissue damage or abnormal osteoclast-mediated bone resorption. Tumor cells and associated inflammatory (immune) cells produce a variety of chemical mediators including prostaglandins (PGE2), nerve growth factor (NGF), endothelins (ET-1) and bradykinin (BK). Several of these proinflammatory mediators have receptors on peripheral terminals and can directly activate or sensitize nociceptors. It is suggested that movement-evoked breakthrough pain in cancer patients is partially due to the tumor-induced loss of the mechanical strength and stability of the tumor-bearing bone so that normally innocuous mechanical stress can now produce distortion of the putative mechanotransducers (TRPV1, TRPV4, and TRPA1) that innervate the bone. 

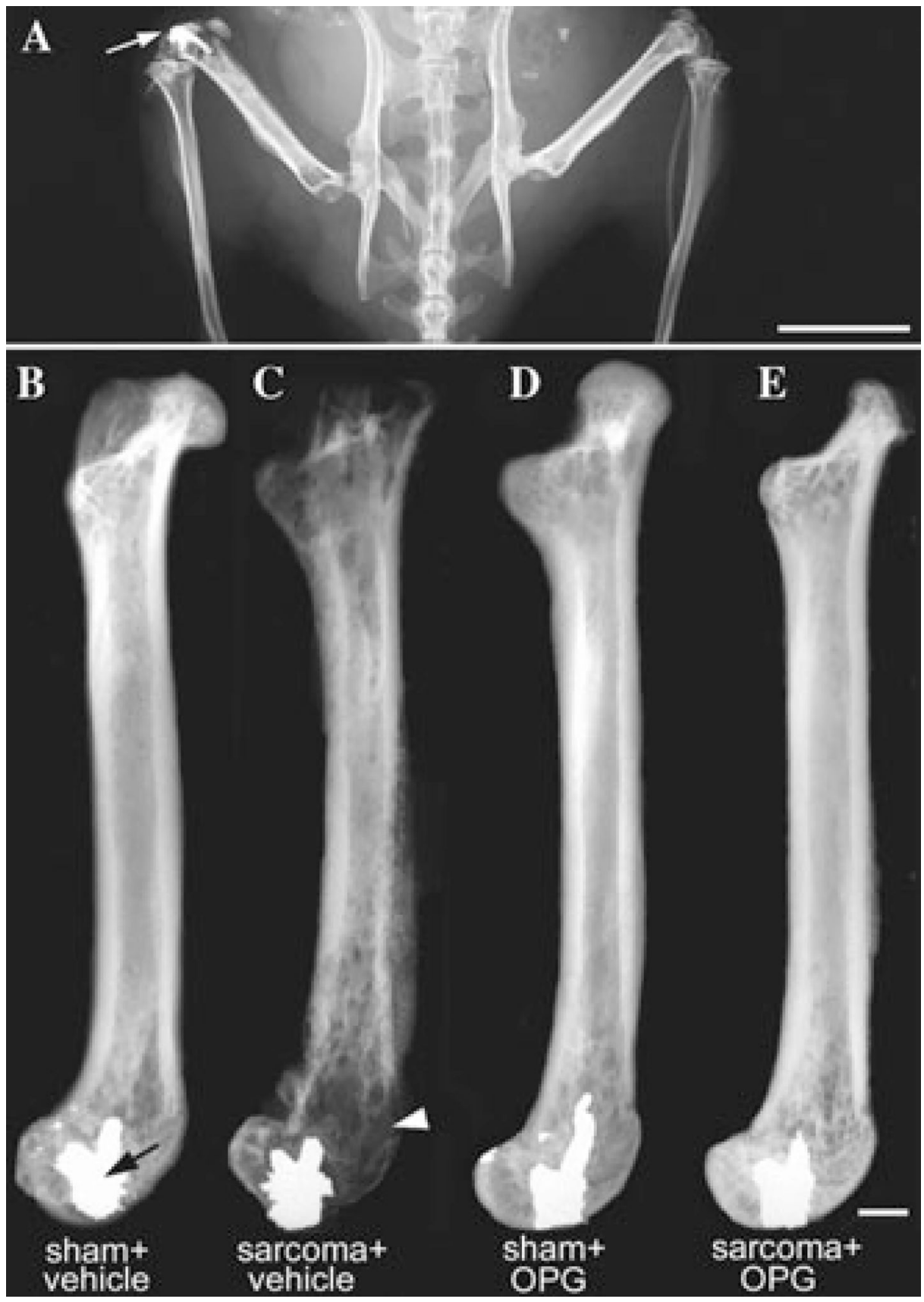

Figure 4.

Osteoprotegerin (OPG) attenuates sarcoma-induced bone destruction in a mouse model of bone cancer pain. (A) Low-power frontal radiograph of mouse pelvis and hind limbs following unilateral injection of 2472 murine osteosarcoma cells into the distal end of the femur and closure of the injection with a dental amalgam plug (arrow). The amalgam plug was used to prevent tumor cells from growing outside the bone. High-resolution radiographs of sham-injected (B and $\mathrm{D}$ ) and sarcoma-injected (C and $\mathrm{E}$ ) femurs from mice that received vehicle (B and C) or OPG (D and E). Note that at day 17 after the injection of the osteosarcoma cells, there is significant bone destruction in the distal femur without OPG (C; 
white arrowhead), whereas tumor induced bone destruction is not evident in sarcomainjected mouse that received OPG (E). Scale bars represent $10 \mathrm{~mm}$ (a) and $0.5 \mathrm{~mm}$ (b-e; bottom panel). (With permission from Honore, P. et al. ${ }^{7}$ ) 
A Spontaneous Guarding

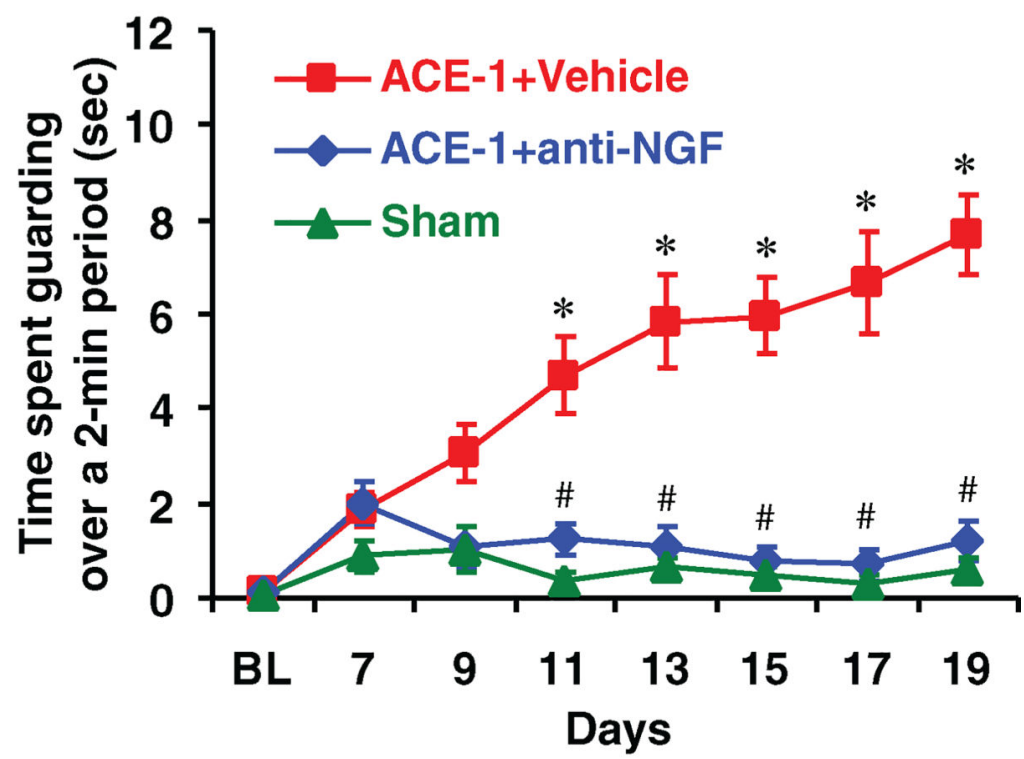

B Spontaneous Flinching

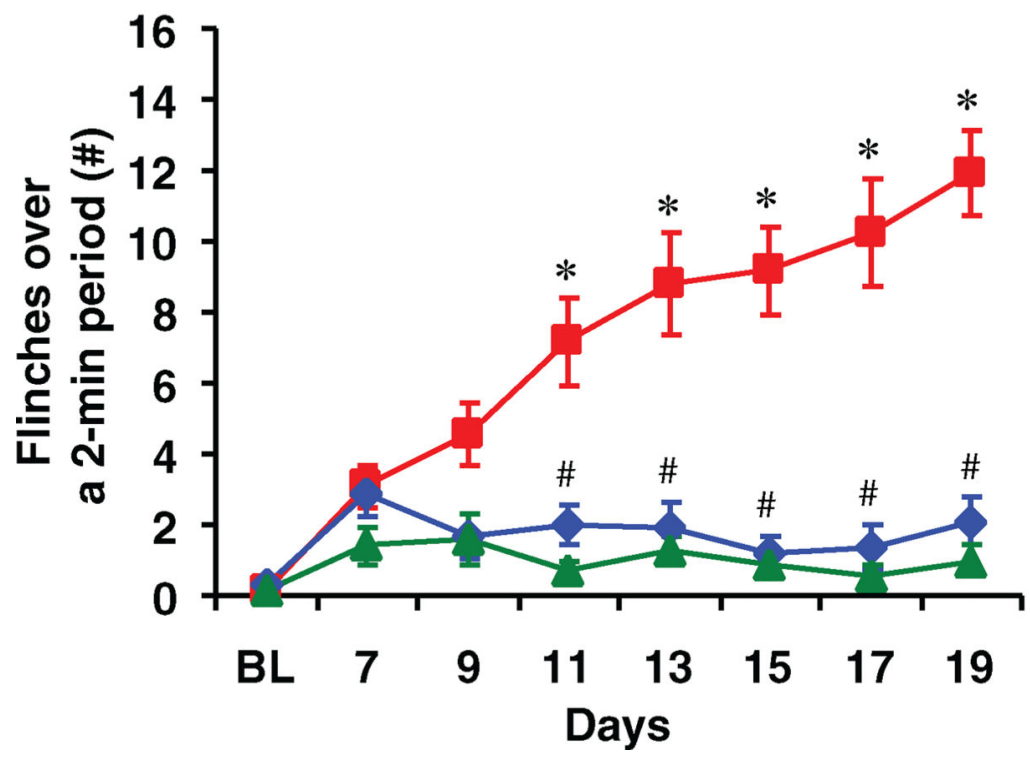

Figure 5.

Anti-NGF attenuates spontaneous bone cancer pain in a model where the tumor cells (canine prostate cells) do not express NGF. Anti-NGF treatment (10 mg/kg, i.p., given on days 7, 12, and 17 posttumor injection) attenuated ongoing bone cancer pain behavior on days 7-19 posttumor injection. In these experiments, canine prostate carcinoma (ACE-1) cells were injected into the femur of adult male mice. The time spent guarding (A) and number of spontaneous flinches (B) of the afflicted limb over a 2-minute observation period were used as measures of ongoing pain. Anti-NGF significantly reduced ongoing pain behaviors in tumor-injected mice as compared with ACE-1 + vehicle. Note that ACE-1 cells in vitro 
express undetectable levels of NGF mRNA or protein, suggesting that NGF could be released mainly from tumor-associated macrophage and immune cells. Bars represent mean S.E.M. $* P<0.05$ versus sham + vehicle; ${ }^{\#} P<0.05$ versus ACE- $1+$ vehicle. (With permission from Halvorson, K.G. et al..$^{10}$ ) 